\title{
Uniform Polarization Scattering With Fiber-Coil-Based Polarization Controllers
}

\author{
Nelson J. Muga, Armando Nolasco Pinto, Member, IEEE, Mário F. S. Ferreira, and \\ José R. Ferreira da Rocha, Member, IEEE
}

\begin{abstract}
A detailed study of fiber-coil-based polarization controllers (PCs) is performed. First, a method to deterministically calculate the $P C$ configuration in order to transform between any two states of polarization is presented. In a second stage, the case in which the configuration angles are randomly changed is studied. The cases of a single PC and of the system obtained with the concatenation of several PCs are analyzed. For both cases, a general expression for the variance of the Stokes parameters is obtained. Using this expression, it is demonstrated that it is possible to achieve uniform polarization scattering using a concatenation of fiber-coil-based PCs. Finally, it is shown that fiber-coil-based PCs can be used to emulate both first- and second-order polarizationmode dispersions.
\end{abstract}

Index Terms-Polarization controllers (PC), polarization-mode dispersion (PMD), polarization scattering.

\section{INTRODUCTION}

$\mathbf{P}$ OLARIZATION control and monitoring are major issues in modern high-speed $(\geq 40 \mathrm{~Gb} / \mathrm{s})$ and long-haul $(\geq 100 \mathrm{~km}$ ) optical communication systems due to the small tolerance of these systems to signal distortion. The first polarization control schemes used in fiber optics communication systems were based on the elastooptic properties of silica by means of controlled squeezing [1]-[4] or bending the fiber itself [5]. Subsequently, other devices were proposed based on electrooptics crystals [6], Faraday rotators [7], and liquid crystals [8], [9]. Nevertheless, the most common polarization controller (PC), at least in laboratorial systems, is the fiber-coilbased PC.

The fiber-coil-based PC uses the elastooptic properties of silica fibers to control the output state of polarization (SOP). Low cost, ease of handling, and the ability to transform between any two SOPs are the main reasons for the widespread use of this PC device. The first PC device using fiber coils was presented by Lefevre in 1980 [5] and patented in 1983 [10].

In [5], Lefevre describes a new single-mode fiber optic device (a fiber coil), which is equivalent to a fractional wave plate

Manuscript received March 30, 2006; revised July 26, 2006. This work was supported in part by the Portuguese Scientific Foundation, FCT, through the "Polarization Mode Dispersion in High-Speed Optical Communication Systems" (PMD) under Project, POSI/CPS/47389/2002, and the FEDER, and POSI programs.

N. J. Muga is with the Physics Department, University of Aveiro and Institute of Telecommunications, 3810-193 Aveiro, Portugal (e-mail: muga@av.it.pt).

A. N. Pinto and J. R. Ferreira da Rocha are with the Department of Electronic and Telecommunications, University of Aveiro and Institute of Telecommunications, 3810-193 Aveiro, Portugal (e-mail: anp@det.ua.pt; frocha@det.ua.pt).

M. F. S. Ferreira is with the Physics Department, University of Aveiro, 3810-193 Aveiro, Portugal (e-mail: mferreira@ fis.ua.pt).

Digital Object Identifier 10.1109/JLT.2006.883642 of classical optics. He also showed how to use a combination of fiber coils to control the polarization inside a single-mode fiber. In the proposed scheme, the fiber was looped into three coils to create three independent fractional wave plates: two quarterwave plates (QWP) and one half-wave plate (HWP). The first QWP transforms the input SOP into a linear polarization. The HWP rotates the linear polarization, and the last QWP transforms the linear polarization into the desired SOP. This device became known as QWP-HWP-QWP fiber-coil-based $\mathrm{PC}$, and this configuration is still prevalent. However, other schemes were proposed (see, for instance, the study in [11]).

In the late 1980s, the studies related with PCs were mainly driven by the need of controlling the SOP in optical receivers for coherent optical transmission systems. A good overview of the work done during that time can be found in [12]. With the advent of erbium-doped fiber amplifiers, the coherent systems became less attractive, and the number of publications related to PCs also decreased. More recently, the study of polarization effects in optical fibers became more intense in connection with the problem of polarization-mode dispersion (PMD), which strongly limits the reach of high-speed optical communication systems.

In this paper, a detailed study of fiber-coil-based PCs is performed. In Section II, a review of the QWP-HWP-QWP fibercoil-based polarization control physics is done. Subsequently, in Section III, we present a mathematical model for this PC. A method that allows the determination of the PC configuration angles to convert between any two states of polarization is presented. In Section IV, we analyze the PC when its configuration is randomly changed. In particular, we investigated the problem of uniformly scattering the light polarization, which is a central problem in the construction of PMD emulators. In Section V, we discuss the possibility of building a PMD emulator based on this simple device. Finally, the main conclusions are presented in Section VI.

\section{Physics of the Fiber-CoIL-B Ased PC}

In single-mode fibers, the birefringence arises from the loss of circular symmetry of the core or by mechanical stress through the elastooptic effect. When a fiber is bent, as shown in Fig. 1, the birefringence is mainly due to mechanical stress [13]. The dominant stress component takes place along the $z$ direction. Nevertheless, it does not contribute directly to the birefringence as it is an odd function of $y$ (is a tensile stress for $y<0$ and a compressive stress for $y>0$ across the $x y$ plane), whereas the electromagnetic modes functions are even 


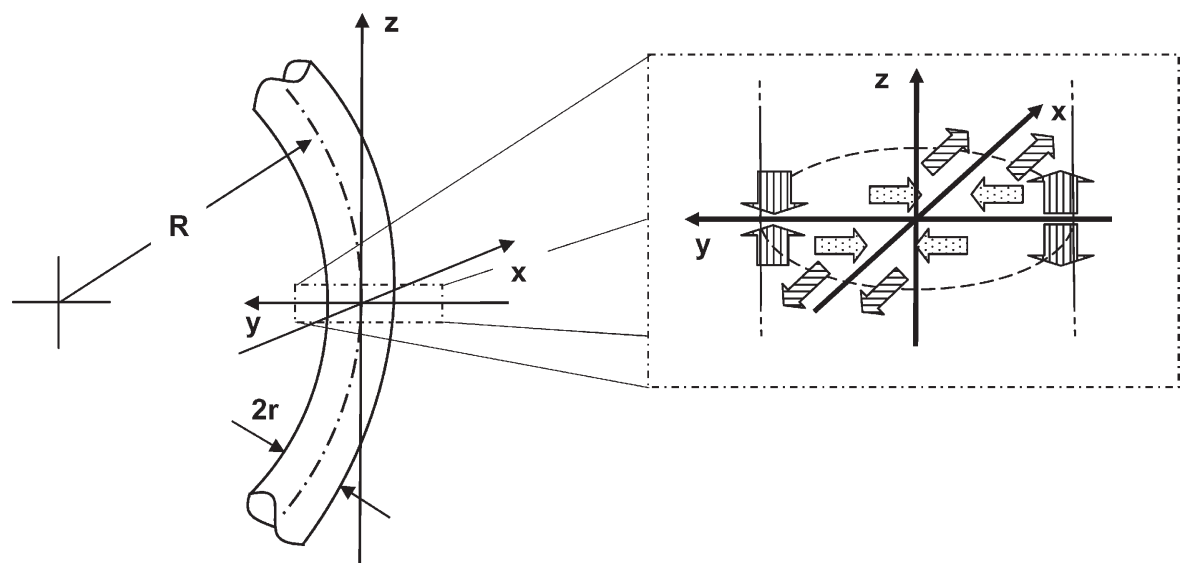

Fig. 1. Bent fiber and its principal mechanical stress.

functions [14]. In fact, the birefringence arises due to the lateral stress in the $x$ and $y$ directions. The lateral stress induces a change in the fiber refractive index along the two main axes of the fiber due to the elastooptic properties of the silica, which are given by [5]

$$
\begin{aligned}
& \Delta n_{x}=\frac{n^{3}}{4}\left(p_{12}-\nu p_{12}-\nu p_{11}\right)\left(\frac{r}{R}\right)^{2} \\
& \Delta n_{y}=\frac{n^{3}}{4}\left(p_{11}-2 \nu p_{12}\right)\left(\frac{r}{R}\right)^{2}
\end{aligned}
$$

where $\nu$ is the Poisson's ratio, $p_{11}$ and $p_{12}$ are two terms of the fiber material photoelasto tensor, and $r$ and $R$ are the fiber and the curvature radius, respectively (see Fig. 1). If we define $\delta n$ as the refractive index difference between the fast and slow axes, we obtain

$$
\delta n=-a\left(\frac{r}{R}\right)^{2}
$$

with $a \simeq 0.133$ [5] for silica fibers. Although the refractive index difference is small, it can produce a considerable phase delay over a distance correspondent to a large number of wavelengths. In fact, by coiling the fiber with a small number of turns with a few centimeters of radius, it is possible to obtain an optical phase difference of $\lambda / m$ between the main polarization axes, where $m$ is a number greater than zero. For $m$ equal to two and four, the HWP and QWP are, respectively, obtained.

In [5], an expression for $R$ as a function of the optical path difference $\lambda / m$ and the number of turns $N$ was obtained

$$
R(\lambda / m, N)=2 \pi a r^{2} \frac{N}{\lambda / m} .
$$

Using (4), it is possible to build a device equivalent to a wave plate of classical optics. However, just like in classical optics, in order to control the light SOP, we must be able to rotate the wave-plate retardation axes in relation to the SOP of the incident light.

In Fig. 2, a fiber coil with a radius $R$ is depicted, where A and $\mathrm{C}$ are two fixed points. With this setup, it is possible to rotate the wave-plate retardation axes in relation to the SOP of the incident light. When the coil plane is rotated through an angle $\theta$, a twist effect is present in $\mathrm{AB}$ and $\mathrm{B}$ 'C, making

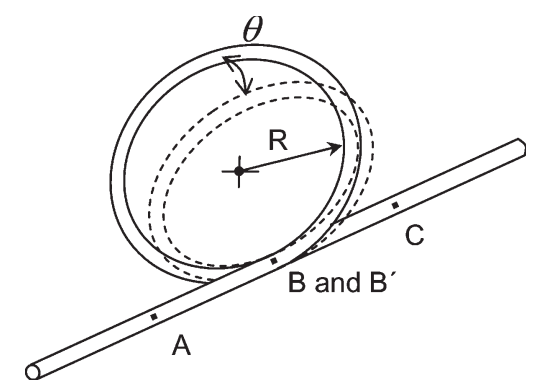

Fig. 2. Schematic representation of a fiber coil. Points $A$ and $C$ are fixed in a lab coordinate system, and $\mathrm{B}$ and $\mathrm{B}^{\prime}$ are fixed in a coordinate system solidary with the fiber coil.

the effective rotation angle equal to $(1-t) \theta$, where $t$ is the twist effect coefficient [5]. According to the study in [14], $t$ takes a small value for silica fibers, approximately equal to 0.08 , making the effective rotation angle almost equal to the physical rotation angle.

In conclusion, a fiber coil can be seen as a classical wave plate, where the phase delay is fixed, and the principal axes orientation are changeable.

\section{MATHEMATiCAL Model FOR THE FIBER-COIL-BASED PC}

A QWP allows us to convert any input SOP into a linear SOP and vice versa, and an HWP allows us to change between any two linear SOPs. To transform an arbitrary input SOP into an arbitrary output SOP as well, the QWP-HWP-QWP wave plate combination is the most commonly used [5]. In the following, a method to deterministically calculate the QWP-HWP-QWP PC configuration (i.e., the three wave-plate angles) for any given input and output SOP is presented.

If we have an input SOP and we aim to generate another SOP at the PC output, we must select the correct configuration angles $\left(\theta_{1}, \theta_{2}\right.$, and $\left.\theta_{3}\right)$ (see Fig. 3). The SOP at the input and output of the PC can be written, respectively, as the Stokes vectors

$$
\hat{s}_{i}=\left[\left(s_{1}\right)_{i},\left(s_{2}\right)_{i},\left(s_{3}\right)_{i}\right]^{\mathrm{T}}
$$

and

$$
\hat{s}_{o}=\left[\left(s_{1}\right)_{o},\left(s_{2}\right)_{o},\left(s_{3}\right)_{o}\right]^{\mathrm{T}}
$$




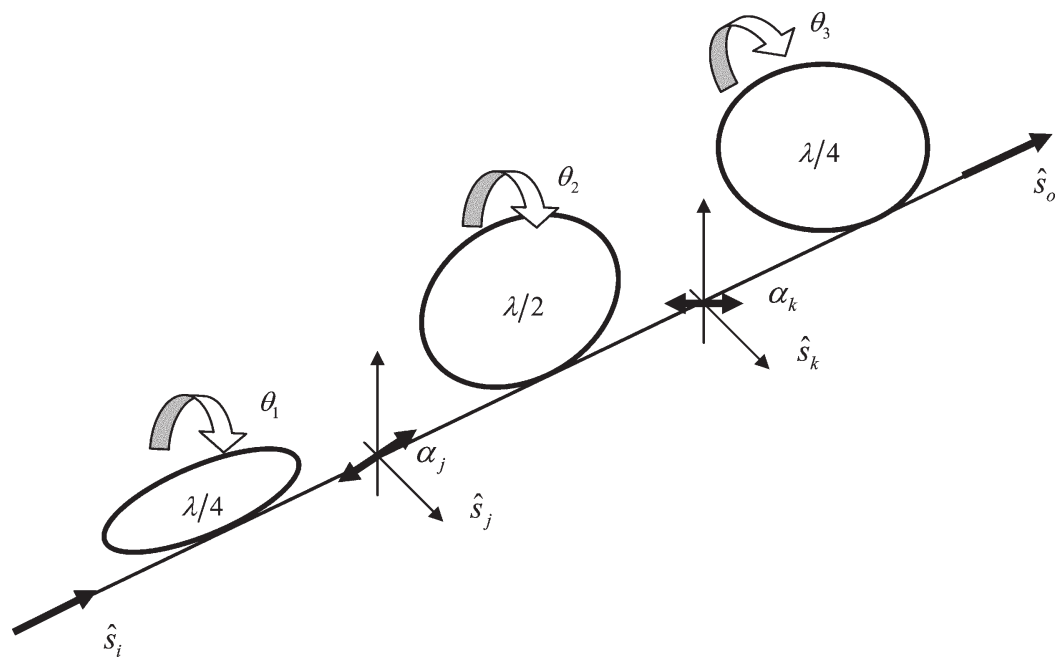

Fig. 3. Schematic representation of the QWP-HWP-QWP wave plates configuration.

where $\mathrm{T}$ indicates the transpose. In the same way, the SOP after the QWP and HWP can be written, respectively, as $\hat{s}_{j}=\left[\left(s_{1}\right)_{j},\left(s_{2}\right)_{j},\left(s_{3}\right)_{j}\right]^{\mathrm{T}}$ and $\hat{s}_{k}=\left[\left(s_{1}\right)_{k},\left(s_{2}\right)_{k},\left(s_{3}\right)_{k}\right]^{\mathrm{T}}$. Therefore, after going through the first QWP, the SOP is given by

$$
\hat{s}_{j}=\mathbf{R}\left(\theta_{1}\right) \mathbf{M}_{\lambda / 4} \mathbf{R}\left(-\theta_{1}\right) \hat{s}_{i}
$$

where $\theta_{1}$ is the angle of the first wave plate, $\mathbf{M}_{\lambda / 4}$ is the QWP matrix, and $\mathbf{R}$ is the rotation matrix of the wave plate. In the Stokes space, the $\mathbf{M}_{\lambda / 4}$ and $\mathbf{R}$ matrices are, respectively, given by

$$
\mathbf{M}_{\lambda / 4}=\left[\begin{array}{ccc}
1 & 0 & 0 \\
0 & 0 & -1 \\
0 & 1 & 0
\end{array}\right]
$$

and

$$
\mathbf{R}=\left[\begin{array}{ccc}
\cos (2 \theta) & -\sin (2 \theta) & 0 \\
\sin (2 \theta) & \cos (2 \theta) & 0 \\
0 & 0 & 1
\end{array}\right]
$$

Equation (7) can therefore be written as (10), shown at the bottom of the page. In order to transform the input SOP $\hat{s}_{i}$ into a linear SOP $\hat{s}_{j}$, we must choose for the first plate (QWP) an angle $\theta_{1}$, such that the third component of (10) vanishes. Therefore, $\theta_{1}$ is given by

$$
\theta_{1}=\frac{1}{2} \arctan \left(\frac{\left(s_{2}\right)_{i}}{\left(s_{1}\right)_{i}}\right)
$$

Under this condition, $\hat{s}_{j}$ can be written as

$$
\hat{s}_{j}=[X, Y, 0]^{\mathrm{T}}
$$

where $X$ and $Y$ are the two non-null Stokes parameters at the input of the second plate (HWP)

$$
\begin{aligned}
X= & \left(s_{1}\right)_{i} \cos ^{2}\left(2 \theta_{1}\right)+\left(s_{2}\right)_{i} \cos \left(2 \theta_{1}\right) \sin \left(2 \theta_{1}\right) \\
& +\left(s_{3}\right)_{i} \sin \left(2 \theta_{1}\right) \\
Y= & \left(s_{1}\right)_{i} \cos \left(2 \theta_{1}\right) \sin \left(2 \theta_{1}\right)+\left(s_{2}\right)_{i} \sin ^{2}\left(2 \theta_{1}\right) \\
& -\left(s_{3}\right)_{i} \cos \left(2 \theta_{1}\right) .
\end{aligned}
$$

Light after the first plate $\left(\hat{s}_{j}\right)$ presents a linear SOP. The second plate will transform it into another linear $\mathrm{SOP}\left(\hat{s}_{k}\right)$ given by

$$
\hat{s}_{k}=[W, Z, 0]^{\mathrm{T}}
$$

where $W$ and $Z$ are functions of the final polarization state $\left(\hat{s}_{o}\right)$. The linear SOPs $\hat{s}_{j}$ and $\hat{s}_{k}$ can be characterized by their respective polarization plane angles $\alpha_{j}$ and $\alpha_{k}$ as

$$
\begin{aligned}
\alpha_{j} & =\frac{1}{2} \arctan \left(\frac{Y}{X}\right) \\
\alpha_{k} & =\frac{1}{2} \arctan \left(\frac{Z}{W}\right) .
\end{aligned}
$$

Considering the situation where $\theta_{2}=0$, the $\hat{s}_{k}$ vector is obtained by multiplying the $\mathbf{M}_{\lambda / 2}$ matrix

$$
\mathbf{M}_{\lambda / 2}=\left[\begin{array}{ccc}
1 & 0 & 0 \\
0 & -1 & 0 \\
0 & 0 & -1
\end{array}\right]
$$

$$
\hat{s}_{j}=\left[\begin{array}{c}
\left(s_{1}\right)_{i} \cos ^{2}\left(2 \theta_{1}\right)+\left(s_{2}\right)_{i} \cos \left(2 \theta_{1}\right) \sin \left(2 \theta_{1}\right)+\left(s_{3}\right)_{i} \sin \left(2 \theta_{1}\right) \\
\left(s_{1}\right)_{i} \cos \left(2 \theta_{1}\right) \sin \left(2 \theta_{1}\right)+\left(s_{2}\right)_{i} \sin ^{2}\left(2 \theta_{1}\right)-\left(s_{3}\right)_{i} \cos \left(2 \theta_{1}\right) \\
\left(s_{2}\right)_{i} \cos \left(2 \theta_{1}\right)-\left(s_{1}\right)_{i} \sin \left(2 \theta_{1}\right)
\end{array}\right]
$$




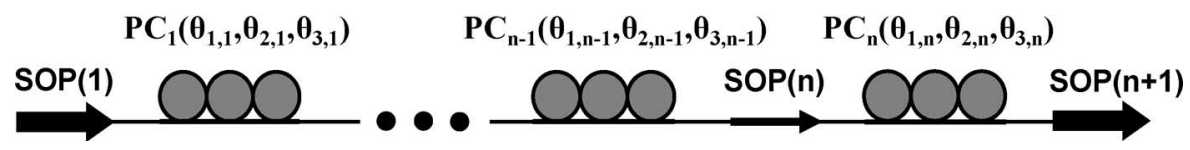

Fig. 4. Schematic representation of $n$ concatenated PCs used for SOP random scattering.

by $\hat{s}_{j}$. Then, we obtain

$$
\hat{s}_{k}=[X,-Y, 0]^{\mathrm{T}} .
$$

From (19), we observe that when $\theta_{2}=0$, the output angle is just the inverse of the input angle $\alpha_{k}=-\alpha_{j}$. It is also known that a $\theta$ rotation of an HWP induces a rotation in the linear polarization of $2 \theta$ [5]. Thus, the polarization plane angle of the light at the output of the second fiber coil is given by

$$
\alpha_{k}=-\alpha_{j}+2 \theta_{2} .
$$

In order to obtain the wave-plate angle $\theta_{2}$, we use (16), (17), and (20)

$$
\theta_{2}=\frac{1}{4}\left[\arctan \left(\frac{Y}{X}\right)+\arctan \left(\frac{Z}{W}\right)\right] .
$$

Note that $\theta_{2}$ is both a function of the input SOP $\hat{s}_{i}$ and output SOP $\hat{s}_{o}$. The angle of the third wave plate $W$ and $Z$ are determined using an equation analogous to (7)

$$
\hat{s}_{o}=\mathbf{R}\left(\theta_{3}\right) \mathbf{M}_{\lambda / 4} \mathbf{R}\left(-\theta_{3}\right) \hat{s}_{k} .
$$

Using again the matrices $\mathbf{M}_{\lambda / 4}$ and $\mathbf{R}$, given by (8) and (9), respectively, we obtain

$$
\left[\begin{array}{l}
\left(s_{1}\right)_{o} \\
\left(s_{2}\right)_{o} \\
\left(s_{3}\right)_{o}
\end{array}\right]=\left[\begin{array}{c}
W \cos ^{2}\left(2 \theta_{3}\right)+Z \cos \left(2 \theta_{3}\right) \sin \left(2 \theta_{3}\right) \\
W \cos \left(2 \theta_{3}\right) \sin \left(2 \theta_{3}\right)+Z \sin ^{2}\left(2 \theta_{3}\right) \\
Z \cos \left(2 \theta_{3}\right)-W \sin \left(2 \theta_{3}\right)
\end{array}\right] .
$$

From (23) and by knowing $\left(s_{1}\right)_{o}$ and $\left(s_{2}\right)_{o}$, we get

$$
\theta_{3}=\frac{1}{2} \arctan \left(\frac{\left(s_{2}\right)_{o}}{\left(s_{1}\right)_{o}}\right) .
$$

With the help of (24) and with the knowledge of $\left(s_{3}\right)_{o}$, we also find expressions for $W$ and $Z$ as

$$
\begin{aligned}
W= & \left(s_{1}\right)_{o} \cos ^{2}\left(2 \theta_{3}\right) \\
& +\left(s_{2}\right)_{o} \cos \left(2 \theta_{3}\right) \sin \left(2 \theta_{3}\right)-\left(s_{3}\right)_{o} \sin \left(2 \theta_{3}\right) \\
Z= & \left(s_{1}\right)_{o} \cos \left(2 \theta_{3}\right) \sin \left(2 \theta_{3}\right) \\
& +\left(s_{2}\right)_{o} \sin ^{2}\left(2 \theta_{3}\right)-\left(s_{3}\right)_{o} \cos \left(2 \theta_{3}\right) .
\end{aligned}
$$

In conclusion, (11), (21), and (24), in conjugation with (5), (6), (13), (14), (25), and (26), provide the three wave-plate angles in order to transform between any two SOP.

Like each individual wave plate, the fiber-coil PC can also be represented by its matrix, resulting from the concatenation of the wave-plate matrices. Therefore, the matrix $\mathbf{F}$ that describes the $\mathrm{PC}$ is given by

$$
\begin{aligned}
\mathbf{F}\left(\theta_{1}, \theta_{2}, \theta_{3}\right)=\mathbf{R}\left(\theta_{3}\right) \mathbf{M}_{\lambda / 4} \mathbf{R}\left(-\theta_{3}\right) \mathbf{R}\left(\theta_{2}\right) \\
\times \mathbf{M}_{\lambda / 2} \mathbf{R}\left(-\theta_{2}\right) \mathbf{R}\left(\theta_{1}\right) \mathbf{M}_{\lambda / 4} \mathbf{R}\left(-\theta_{1}\right) .
\end{aligned}
$$

Matrix $\mathbf{F}$ can be used to calculate the output SOP $\hat{s}_{o}$, given the input SOP $\hat{s}_{i}$, and the configuration angles $\left(\theta_{1}, \theta_{2}\right.$, and $\left.\theta_{3}\right)$.

\section{RANDOM Distribution OVER THE POINCARÉ SPHERE}

In order to test the ability to uniformly scatter an input SOP over the Poincaré sphere, we use a system resulting from the concatenation of several fiber-based PCs, as schematically represented in Fig. 4. A device or a concatenation of devices, with the ability to produce a uniform scatter over the Poincaré sphere, can be useful, for instance, in the development of PMD emulators. Indeed, some authors have proposed PMD emulators, based on pieces of polarization-maintaining fibers (PMFs) interconnected with uniform scattering devices [15], [16].

To verify the scattering uniformity, the statistics of the three Stokes parameters must be evaluated and compared with the theoretical predictions. As derived in the Appendix, if we have a uniform distribution of points over the Poincare sphere, each Stokes vector component $s_{i}$ has a mean value equal to zero, variance of one third, and uniform distribution between -1 and 1 . The procedure used to investigate the evolution of the polarization scattering of our device, namely the variance convergence to one third, was inspired in the Marcuse analysis of two rotation matrices [17].

\section{A. Theoretical Derivation}

As can be seen in Fig. 4, the output signal of a PC is used as the input of the next PC. The SOP statistics at the end of each PC is evaluated and compared with the desired uniform distribution. The number of concatenated elements is increased in order to improve the scattering uniformity.

The SOP at the $n$th PC output is related to the SOP at the $n$th $\mathrm{PC}$ input by the following expression:

$$
\left[\begin{array}{c}
\left(s_{1}\right)_{n+1} \\
\left(s_{2}\right)_{n+1} \\
\left(s_{3}\right)_{n+1}
\end{array}\right]=\mathbf{F}_{n}\left(\theta_{1, n}, \theta_{2, n}, \theta_{3, n}\right)\left[\begin{array}{c}
\left(s_{1}\right)_{n} \\
\left(s_{2}\right)_{n} \\
\left(s_{3}\right)_{n}
\end{array}\right]
$$

where $\mathbf{F}_{n}\left(\theta_{1, n}, \theta_{2, n}, \theta_{3, n}\right)$ is given by (27). The index $n$ was introduced in $\mathbf{F}_{n}\left(\theta_{1, n}, \theta_{2, n}, \theta_{3, n}\right)$ in order to make it clear that the matrix $\mathbf{F}$ and the angles $\theta_{1}, \theta_{2}$, and $\theta_{3}$ refer to the $n$th PC controller. In (28), $\left(s_{i}\right)_{n}$ is the $i$ component of the Stokes vector at the input of the $n$th PC.

We are going to assume that the $\mathrm{PC}$ angles are randomly changed following a uniform distribution between $-\pi$ and $\pi$. We are also going to assume that the change is independent between the angles of the same PCs and between the angles of different PCs. Therefore, the average value of the $\mathbf{F}$ matrix elements $f_{i j}$ will be equal for all the PCs and will be hereafter designated simply by $\left\langle f_{i j}\right\rangle$. 
The mean value of each Stokes parameter at the output of the $n$th $\mathrm{PC}\left\langle\left(s_{i}\right)_{n+1}\right\rangle$ is given by

$$
\left[\begin{array}{c}
\left\langle\left(s_{1}\right)_{n+1}\right\rangle \\
\left\langle\left(s_{2}\right)_{n+1}\right\rangle \\
\left\langle\left(s_{3}\right)_{n+1}\right\rangle
\end{array}\right]=\left[\begin{array}{ccc}
\left\langle f_{11}\right\rangle & \left\langle f_{12}\right\rangle & \left\langle f_{13}\right\rangle \\
\left\langle f_{21}\right\rangle & \left\langle f_{22}\right\rangle & \left\langle f_{23}\right\rangle \\
\left\langle f_{31}\right\rangle & \left\langle f_{32}\right\rangle & \left\langle f_{33}\right\rangle
\end{array}\right]\left[\begin{array}{c}
\left\langle\left(s_{1}\right)_{n}\right\rangle \\
\left\langle\left(s_{2}\right)_{n}\right\rangle \\
\left\langle\left(s_{3}\right)_{n}\right\rangle
\end{array}\right] .
$$

Note that the $n$ index was dropped in the matrix coefficients (29) because all the PCs are, statistically, equivalents. In (29), the mean value $\left\langle f_{11}\right\rangle$, for instance, is calculated by

$$
\left\langle f_{11}\right\rangle=\int_{-\pi}^{\pi} \int_{-\pi}^{\pi} \int_{-\pi}^{\pi} p_{\theta}^{3} f_{11} d \theta_{1} d \theta_{2} d \theta_{3}
$$

where $p_{\theta}$ is the probability density function (pdf) for each wave-plate angle, and $f_{11}$ is obtained directly from (27) and is given by

$$
\begin{aligned}
f_{11}= & 2 \cos ^{2}\left(2 \theta_{1}\right) \cos ^{2}\left(2 \theta_{3}\right) \cos ^{2}\left(2 \theta_{2}\right) \\
& +2 \cos ^{2}\left(2 \theta_{1}\right) \cos \left(2 \theta_{2}\right) \cos \left(2 \theta_{3}\right) \sin \left(2 \theta_{3}\right) \sin \left(2 \theta_{2}\right) \\
& -\cos ^{2}\left(2 \theta_{1}\right) \cos ^{2}\left(2 \theta_{3}\right) \\
& +2 \cos \left(2 \theta_{1}\right) \sin \left(2 \theta_{1}\right) \sin \left(2 \theta_{2}\right) \cos ^{2}\left(2 \theta_{3}\right) \cos \left(2 \theta_{2}\right) \\
& +\cos \left(2 \theta_{1}\right) \sin \left(2 \theta_{1}\right) \cos \left(2 \theta_{3}\right) \sin \left(2 \theta_{3}\right) \\
& -2 \cos \left(2 \theta_{1}\right) \sin \left(2 \theta_{1}\right) \cos \left(2 \theta_{3}\right) \sin \left(2 \theta_{3}\right) \cos ^{2}\left(2 \theta_{2}\right) \\
& +\sin \left(2 \theta_{3}\right) \sin \left(2 \theta_{1}\right)
\end{aligned}
$$

Assuming a uniform distribution between $-\pi$ and $\pi$ for the configuration angles, the pdf is given by $p_{\theta}=1 / 2 \pi$. Using this value in (30) and performing the triple integral, we find that

$$
\left\langle f_{11}\right\rangle=0 \text {. }
$$

Repeating the same proceeding for all the matrix $\mathbf{F}$ elements, we found that the remaining matrix elements have the same average value, $\left\langle f_{i j}\right\rangle=0$; then, we can conclude that, independent

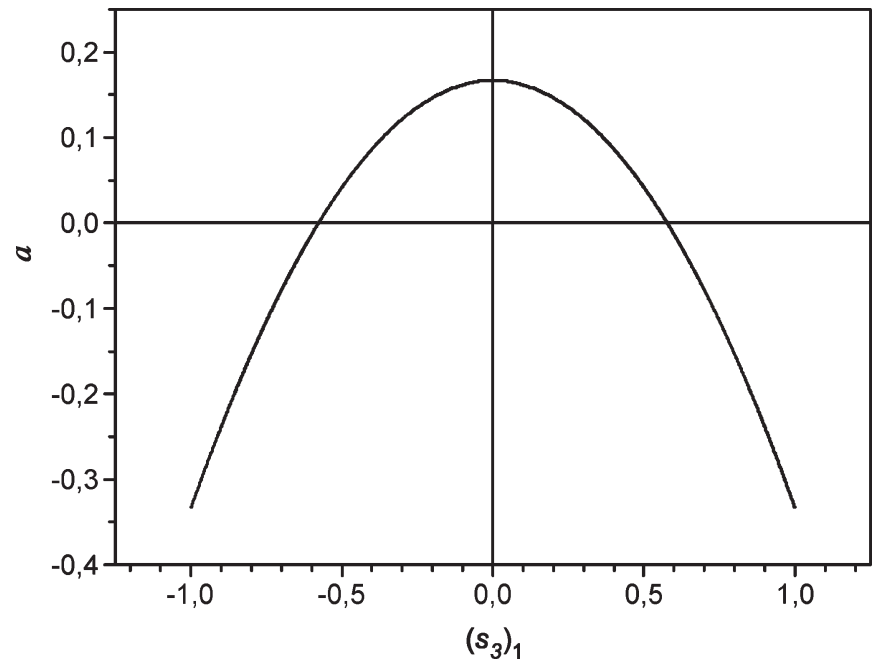

Fig. 5. Parameter $a$ as a function of the third Stokes vector component $\left(s_{3}\right)_{1}$.

of the number of concatenated PCs, each Stokes vector component always has a null average, provided that the configuration angles are uniformly distributed between $-\pi$ and $\pi$.

In order to calculate the variance of each Stokes vector component, we start by squaring each component of (28). Equation (33), shown at the bottom of the page, is obtained. Considering the statistical independence of $\left(s_{i}\right)_{n}$, Stokes vector components at the input of the $n$th PC, and $f_{i j}$ matrix elements of the $n$th PC, the mean values $\left\langle\left(s_{i}\right)_{n+1}^{2}\right\rangle$ can be written as (34), shown at the bottom of the page. The mean values $\left\langle f_{k i} f_{k j}\right\rangle$ are evaluated using an equation analogous to (30)

$$
\left\langle f_{k i} f_{k j}\right\rangle=\int_{-\pi}^{\pi} \int_{-\pi}^{\pi} \int_{-\pi}^{\pi} p_{\theta}^{3} f_{k i} f_{k j} d \theta_{1} d \theta_{2} d \theta_{3}
$$

where $f_{k i}$ and $f_{k j}$ are directly obtained from (27), and $p_{\theta}=1 / 2 \pi$. After calculating the triple integral, we reach the

$$
\left[\begin{array}{l}
\left(s_{1}\right)_{n+1}^{2} \\
\left(s_{2}\right)_{n+1}^{2} \\
\left(s_{3}\right)_{n+1}^{2}
\end{array}\right]=\left[\begin{array}{l}
f_{11, n}^{2}\left(s_{1}\right)_{n}^{2}+f_{12, n}^{2}\left(s_{2}\right)_{n}^{2}+f_{13, n}^{2}\left(s_{3}\right)_{n}^{2}+2 \\
f_{21, n}^{2}\left(s_{1}\right)_{n}^{2}+f_{22, n}^{2}\left(s_{2}\right)_{n}^{2}+f_{23, n}^{2}\left(s_{3}\right)_{n}^{2}+2 \\
\sum_{i, j=1(i \neq j)}^{3} f_{1 i, n} f_{1 j, n}\left(s_{i}\right)_{n}\left(s_{j}\right)_{n} \\
f_{31, n}^{2}\left(s_{1}\right)_{n}^{2}+f_{32, n}^{2}\left(s_{2}\right)_{n}^{2}+f_{33, n}^{2}\left(s_{3}\right)_{n}^{2}+2 f_{2 j, n}\left(s_{i}\right)_{n}\left(s_{j}\right)_{n} \\
\sum_{i, j=1(i \neq j)}^{3} f_{3 i, n} f_{3 j, n}\left(s_{i}\right)_{n}\left(s_{j}\right)_{n}
\end{array}\right]
$$

$$
\left[\begin{array}{l}
\left\langle\left(s_{1}\right)_{n+1}^{2}\right\rangle \\
\left\langle\left(s_{2}\right)_{n+1}^{2}\right\rangle \\
\left\langle\left(s_{3}\right)_{n+1}^{2}\right\rangle
\end{array}\right]=\left[\begin{array}{l}
\left\langle f_{11}^{2}\right\rangle\left\langle\left(s_{1}\right)_{n}^{2}\right\rangle+\left\langle f_{12}^{2}\right\rangle\left\langle\left(s_{2}\right)_{n}^{2}\right\rangle+\left\langle f_{13}^{2}\right\rangle\left\langle\left(s_{3}\right)_{n}^{2}\right\rangle+2 \sum_{i, j=1(i \neq j)}^{3}\left\langle f_{1 i} f_{1 j}\right\rangle\left\langle\left(s_{i}\right)_{n}\left(s_{j}\right)_{n}\right\rangle \\
\left\langle f_{21}^{2}\right\rangle\left\langle\left(s_{1}\right)_{n}^{2}\right\rangle+\left\langle f_{22}^{2}\right\rangle\left\langle\left(s_{2}\right)_{n}^{2}\right\rangle+\left\langle f_{23}^{2}\right\rangle\left\langle\left(s_{3}\right)_{n}^{2}\right\rangle+2 \sum_{i, j=1(i \neq j)}^{3}\left\langle f_{2 i} f_{2 j}\right\rangle\left\langle\left(s_{i}\right)_{n}\left(s_{j}\right)_{n}\right\rangle \\
\left\langle f_{31}^{2}\right\rangle\left\langle\left(s_{1}\right)_{n}^{2}\right\rangle+\left\langle f_{32}^{2}\right\rangle\left\langle\left(s_{2}\right)_{n}^{2}\right\rangle+\left\langle f_{33}^{2}\right\rangle\left\langle\left(s_{3}\right)_{n}^{2}\right\rangle+2 \sum_{i, j=1(i \neq j)}^{3}\left\langle f_{3 i} f_{3 j}\right\rangle\left\langle\left(s_{i}\right)_{n}\left(s_{j}\right)_{n}\right\rangle
\end{array}\right]
$$




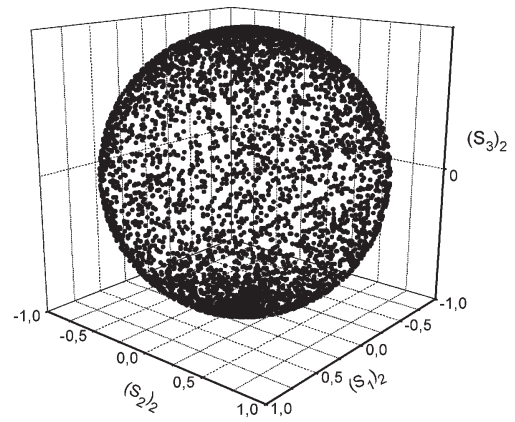

(a)

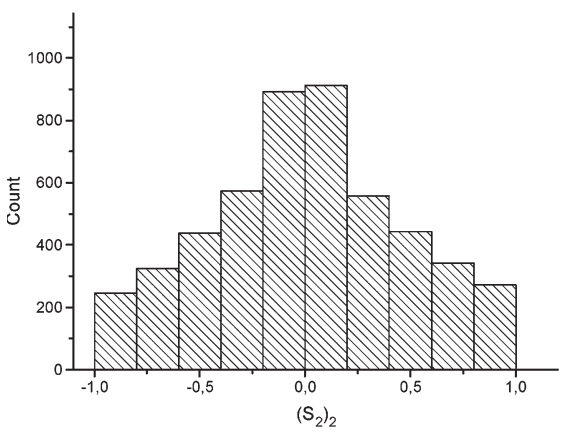

(c)
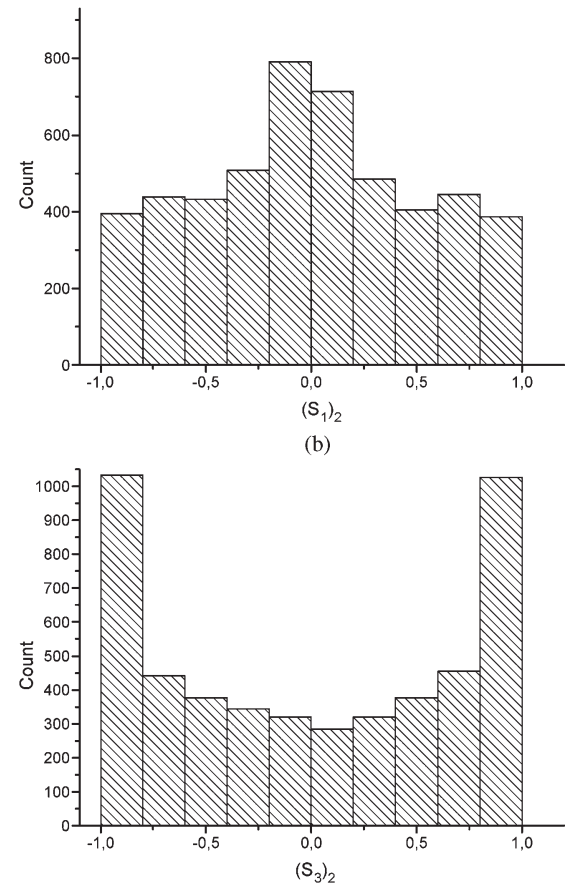

(d)

Fig. 6. Stokes parameters resulting from the scattering due to one PC, considering the initial SOP $\hat{s}_{1}=[0,0,1]^{\mathrm{T}}$. (a) Poincare sphere representation of the output SOP. (b) $\left(s_{1}\right)_{2}$ histogram. (c) $\left(s_{2}\right)_{2}$ histogram. (d) $\left(s_{3}\right)_{2}$ histogram.

conclusion that $\left\langle f_{k i} f_{k j}\right\rangle=0$ when $i \neq j$. Using this result and calculating the values for the case $i=j$, the following expression is obtained:

$$
\left[\begin{array}{c}
\left\langle\left(s_{1}\right)_{n+1}^{2}\right\rangle \\
\left\langle\left(s_{2}\right)_{n+1}^{2}\right\rangle \\
\left\langle\left(s_{3}\right)_{n+1}^{2}\right\rangle
\end{array}\right]=\left[\begin{array}{lll}
3 / 8 & 3 / 8 & 1 / 4 \\
3 / 8 & 3 / 8 & 1 / 4 \\
1 / 4 & 1 / 4 & 1 / 2
\end{array}\right]\left[\begin{array}{c}
\left\langle\left(s_{1}\right)_{n}^{2}\right\rangle \\
\left\langle\left(s_{2}\right)_{n}^{2}\right\rangle \\
\left\langle\left(s_{3}\right)_{n}^{2}\right\rangle
\end{array}\right] .
$$

Equation (36) gives a relation between Stokes vector component variances at the input and output of the $n$th PC. By using (36) iteratively and considering an initial SOP $\hat{s}_{1}=$ $\left[\left(s_{1}\right)_{1},\left(s_{2}\right)_{1},\left(s_{3}\right)_{1}\right]^{\mathrm{T}}$, the following expression is found:

$$
\left[\begin{array}{l}
\left\langle\left(s_{1}\right)_{n+1}^{2}\right\rangle \\
\left\langle\left(s_{2}\right)_{n+1}^{2}\right\rangle \\
\left\langle\left(s_{3}\right)_{n+1}^{2}\right\rangle
\end{array}\right]=\frac{1}{3}\left[\begin{array}{l}
1 \\
1 \\
1
\end{array}\right]+\frac{a}{4^{n}}\left[\begin{array}{c}
1 \\
1 \\
-2
\end{array}\right]+\delta_{n 0} b\left[\begin{array}{c}
1 \\
-1 \\
0
\end{array}\right]
$$

where $\delta_{n 0}$ is the Kronecker symbol, and $a$ and $b$ are functions of $\hat{s}_{1}$

$$
\begin{aligned}
a & =\frac{1}{2}\left(\frac{1}{3}-\left(s_{3}\right)_{1}^{2}\right) \\
b & =\frac{1}{2}\left(\left(s_{1}\right)_{1}^{2}-\left(s_{2}\right)_{1}^{2}\right) .
\end{aligned}
$$

From (37), we verify that the variance of each component of the Stokes vector converges to the one third value with a factor $a / 4^{n}$. Indeed, from (38), we can say that SOPs with the same modulus of $\left(s_{3}\right)_{1}$ have the same convergence rate (note that these SOP domains depict a circumference in the Poincaré sphere parallel to the $s_{3}=0$ plane) and that the convergence rate decreases with the increase of the modulus of $a$. Fig. 5 represents the parameter $a$ versus $\left(s_{3}\right)_{1}$. The parameter $a$ becomes

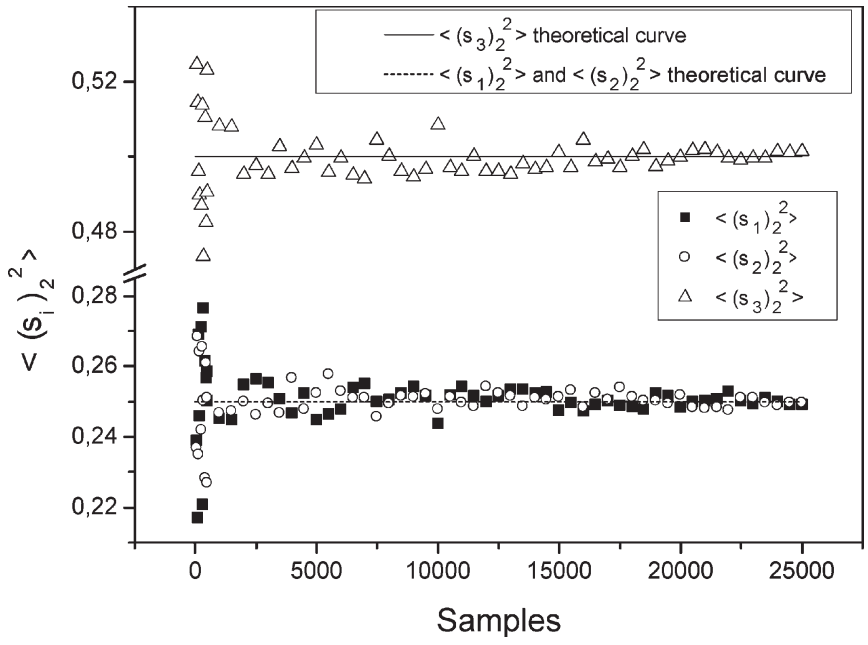

Fig. 7. Mean square of the Stokes parameters as function of the samples number for the case of one PC and $\hat{s}_{1}=[0,0,1]^{\mathrm{T}}$.

null when $\left(s_{3}\right)_{1}^{2}=1 / 3$, which means that, independent of the number of PCs, $\hat{s}_{1}=\left[\left(s_{1}\right)_{1},\left(s_{2}\right)_{1}, \pm \sqrt{1 / 3}\right]^{\mathrm{T}}$ is a special initial SOP. In fact, with this initial SOP, the variance of the Stokes parameters reaches the one third value right after the first PC.

\section{B. Numerical Simulations}

We now present some results, considering the polarization scattering corresponding to different initial SOPs and number of PCs. First, only one PC is considered; subsequently, the number of PCs is increased up to 20.

In Fig. 6(a), 5000 Stokes vectors are represented and obtained at the end of a single PC, considering the same input $\hat{s}_{1}=[0,0,1]^{\mathrm{T}}$ and varying the configuration angles uniformly 


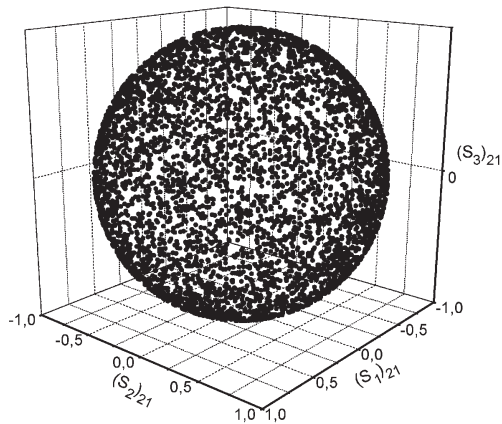

(a)

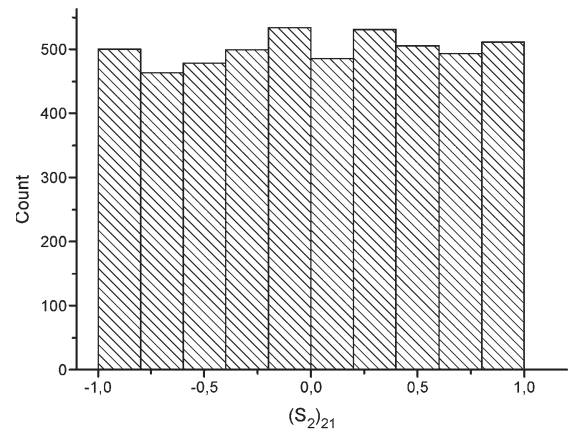

(c)

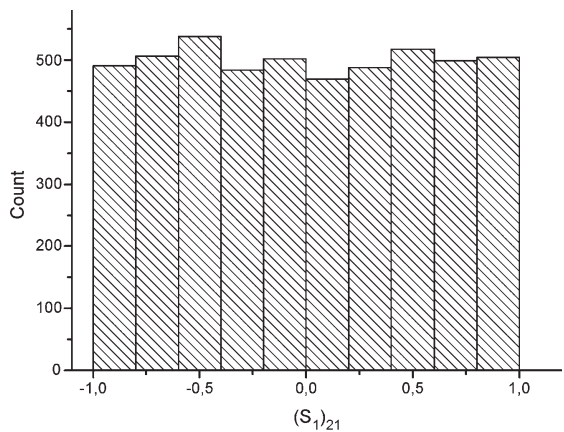

(b)

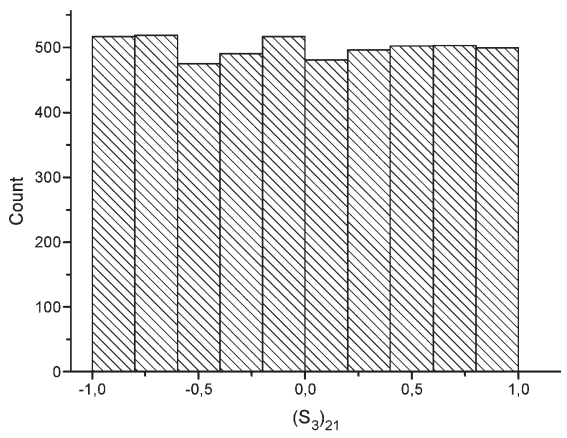

(d)

Fig. 8. Stokes parameters resulting from the scattering due to $20 \mathrm{PCs}$, considering the initial SOP $\hat{s}_{1}=[0,0,1]^{\mathrm{T}}$. (a) Poincaré sphere representation of the output SOP. (b) $\left(s_{1}\right)_{21}$ histogram. (c) $\left(s_{2}\right)_{21}$ histogram. (d) $\left(s_{3}\right)_{21}$ histogram.

between $-\pi$ and $\pi$. As can be seen in Fig. 6(b), (c), and (d), light is not uniformly scattered at the PC output. This result is in accordance with the theoretical prediction. Using (37), the variances for the Stokes parameters at the PC output are given by

$$
\left[\begin{array}{l}
\left\langle\left(s_{1}\right)_{2}^{2}\right\rangle \\
\left\langle\left(s_{2}\right)_{2}^{2}\right\rangle \\
\left\langle\left(s_{3}\right)_{2}^{2}\right\rangle
\end{array}\right]=\left[\begin{array}{c}
\frac{1}{4} \\
\frac{1}{4} \\
\frac{1}{2}
\end{array}\right] .
$$

The numerical results converge for the theoretical values (40) as the numbers of trials increases, as shown in Fig. 7.

This result confirms that one PC does not produce a uniform distribution over the Poincaré sphere. We repeated the same numerical simulations for different polarization states of input light, and we observed that with one PC, the output Stokes parameters' mean-square values are strongly dependent on the initial SOP. Nevertheless, using three different input SOPs, with equal $\left|\left(s_{3}\right)_{1}\right|$, three similar distributions were obtained. This result is in good agreement with the $\left|\left(s_{3}\right)_{1}\right|$ parameter degeneration presented in (37) and (38).

Fig. 8(a) represents 5000 Stokes vectors for an input SOP $\hat{s}_{1}=[0,0,1]^{T}$ using 20 PCs. Fig. 8(b), (c), and (d) shows that the three Stokes parameters are uniformly distributed between -1 and 1 .

According to (37), for large $n$, the mean value of $s_{1}^{2}, s_{2}^{2}$, and $s_{3}^{2}$ converges to one third which is independent of the input SOP. We present in Fig. 9 the mean squares of the Stokes parameters as a function of the PC number to a particular input SOP $\hat{s}_{1}=[0,0,1]^{\mathrm{T}}$. As expected, all mean-square values converge to the one third value.

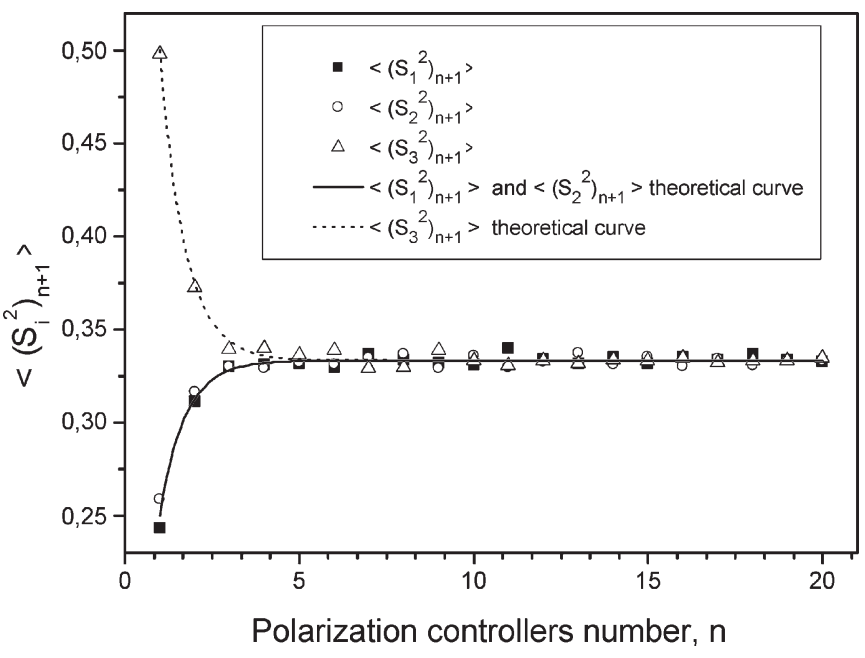

Fig. 9. Mean square of the Stokes parameters as function of PC number, considering the initial SOP $\hat{s}_{1}=[0,0,1]^{\mathrm{T}}$. Numerical values and theoretical curves are shown.

\section{IMPORTANCE OF UNIFORM POLARIZATION SCATTERING IN PMD EMULATION}

We consider in this section an application of the uniform polarization scattering using fiber-coil-based PCs to PMD emulation.

When this paper was about to be finished and without any relation with this paper, results were published [16] that propose a PMD emulator based on a QWP-HWP-QWP fiber-coil-based PC. Even if the analysis presented here is not directly applied to the emulator presented in [16], due to the fact that we assume statistical independence between PCs and, in [16], only a PC 
is used, that work confirms the feasibility of building a PMD emulator based on this simple device.

We consider a system composed of several PMFs with one or more PCs between adjacent PMFs to scatter the light polarization. Each PMF is described by the matrix [15]

$$
\mathbf{R}_{x}=\left[\begin{array}{ccc}
1 & 0 & 0 \\
0 & \cos (2 \phi) & -\sin (2 \phi) \\
0 & \sin (2 \phi) & \cos (2 \phi)
\end{array}\right]
$$

where $\phi=\omega \Delta \tau_{s}$ is the birefringence-induced phase mismatch directly related to the differential group delay (DGD) of the PMF $\Delta \tau_{s}$, and $\omega$ is the angular frequency of the light. The polarization scattering between PMFs can be modeled as

$$
\mathbf{R}_{s}=\prod_{n=1}^{m} \mathbf{F}_{n}\left(\theta_{1, n}, \theta_{2, n}, \theta_{3, n}\right)
$$

where $\mathbf{F}$ is given by (27), and $m$ is the number of PCs used to scatter the light polarization.

\section{A. System With Two PMFs}

In order to assess the effect of the polarization-scattering uniformity, we simulated three systems resulting from the concatenation of two PMFs, with DGD equal to $12 \mathrm{ps}$ and with a polarization-scattering section between PMFs. The polarization-scattering section comprises one PC in the first case, two in the second, and three in the third. The magnitude of the resulting first-order PMD vector (the DGD) was obtained using the matrices presented in (41) and (42) and the wellknown concatenation rule of PMD vectors [18], [19]. The simulation results for each system are represented in Fig. 10, where $10^{5}$ realizations were performed. The three considered cases are presented, respectively, in Fig. 10(a)-(c). Note that each realization is related with an ensemble of three angles $\theta_{1}, \theta_{2}$, and $\theta_{3}$ randomly chosen for each PC. The angles are uniformly distributed between $-\pi$ and $\pi$ and are statistically independent.

For two PMFs fibers with an ideal uniform polarization scattering between them, Djupsjobacka [20] has proven that the pdf for the magnitude of the resulting first-order PMD vector $\Delta \tau$ is given by

$$
f(\Delta \tau)=\frac{\Delta \tau}{2 \Delta \tau_{s}^{2}}, \quad \Delta \tau \in\left[0,2 \Delta \tau_{s}\right]
$$

where $\Delta \tau_{s}$ is the DGD of each single PMF. Comparing our simulation results with (43), represented in Fig. 10 with a solid line, we observe that as the number of PCs used to scatter the polarization increases, the simulation points are closer to the expected theoretical value. We can say that the three PCs used in the simulation depicted in Fig. 10(c) provide a uniform polarization scattering over the Poincare sphere. This result corroborates the one obtained in the last section, where we have shown that the uniformity of the polarization scattering increases with the number of PCs, and, with three PCs, the

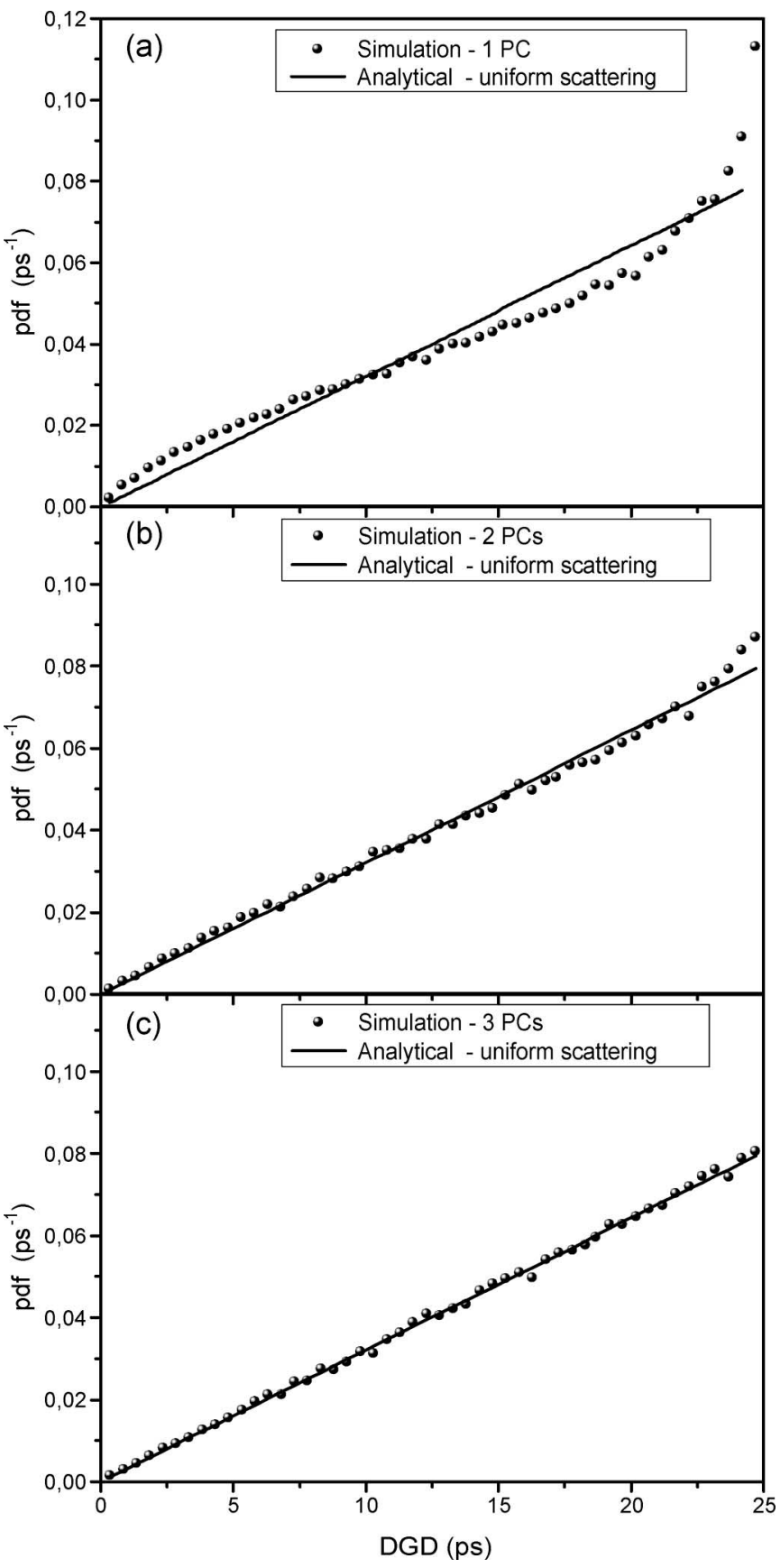

Fig. 10. Pdf for the DGD in a system with two equal-length PMFs, using between them (a) one, (b) two, and (c) three PCs to scatter the light polarization. Solid lines represent the theoretical values, given by (43), and marks represent values obtained from the numerical simulations.

variance of each Stokes vector component approach the one third value [see (37) and Fig. 9].

\section{B. Emulator Based on PMFs and Fiber-Coil-Based PCs}

In this section, we investigate the ability to produce accurate first- and second-order PMD statistics using PMFs and fiber-coil-based PCs. We are going to use $n$ PMFs and $n-1$ polarization-scattering sections.

In a first stage, between PMFs, a light-scattering section comprising three PCs is placed. As we saw previously, the 

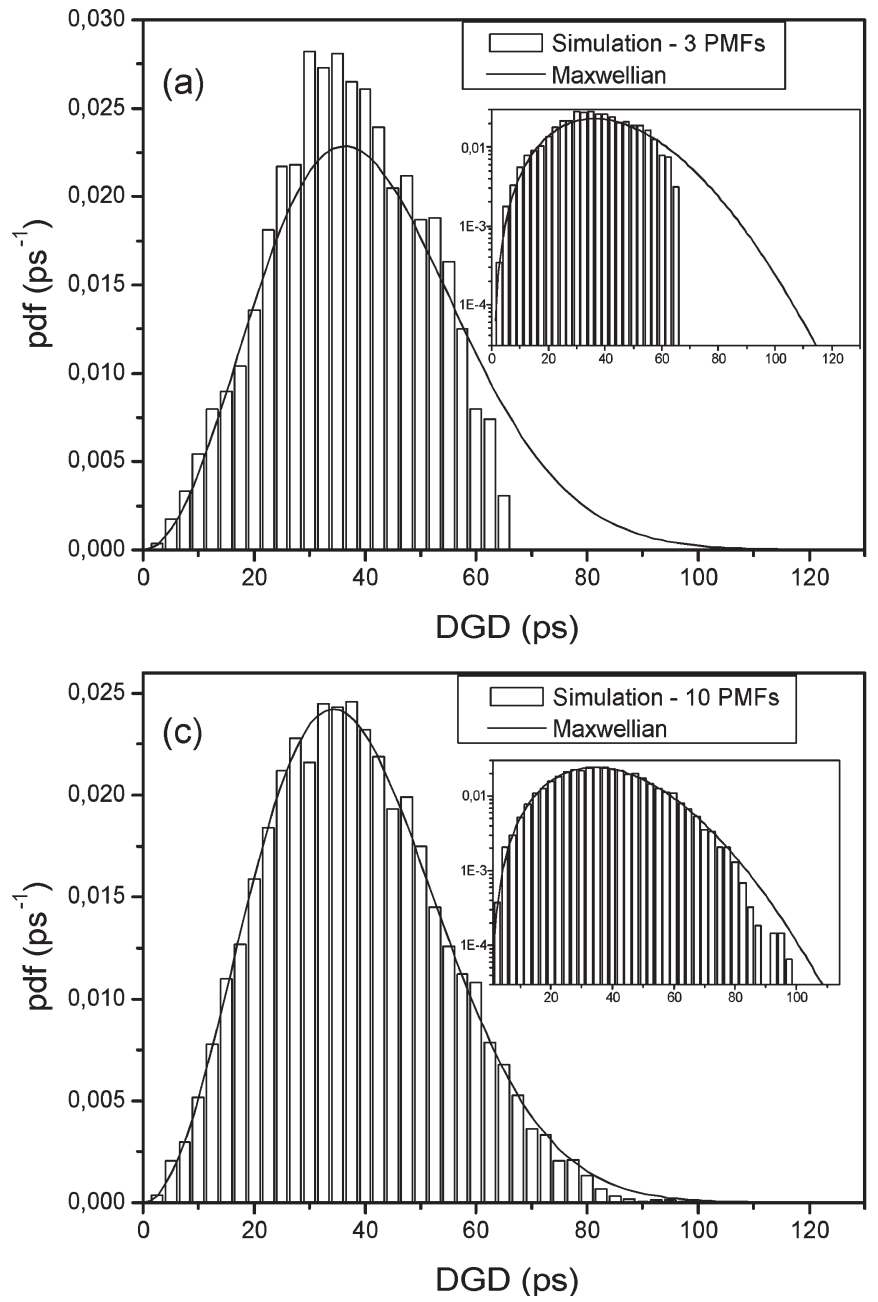
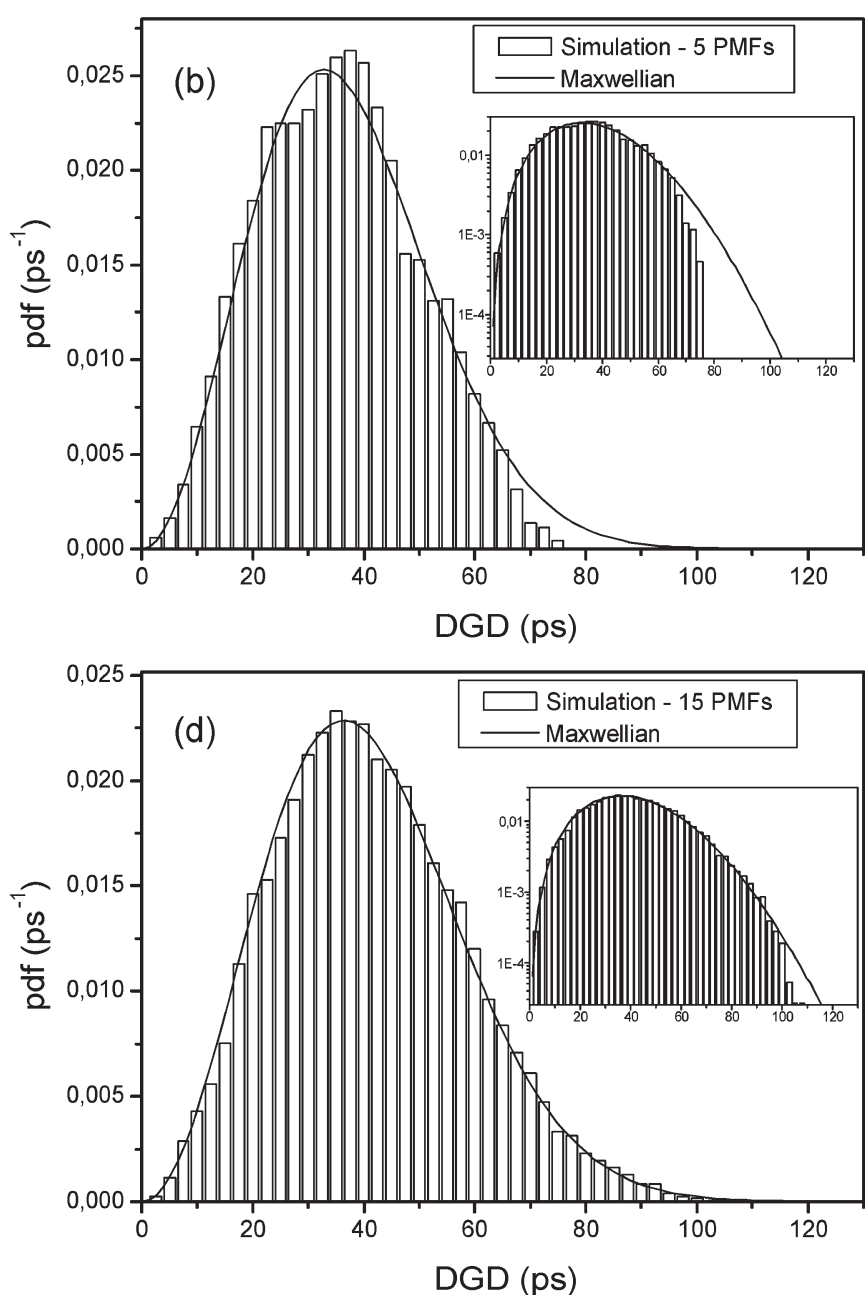

Fig. 11. Pdfs of the first-order PMD vector obtained when a polarization-scattering section comprising three PCs was used in a system with (a) three; (b) five; (c) ten; and (d) 15 PMFs. The Maxwellian is represented as a solid line. Insets show the distributions in a logarithmic scale.

three PCs produce a more uniform scattering over the Poincaré sphere. PMD vectors were simulated using the Müller matrix method, with a 0.025 -nm step and a 0.0083 -nm interleave step [21]. We considered 120 different wavelengths, and for each wavelength, 250 statistically independent combinations for the PCs configuration angles were applied. The length of PMFs was obtained from a Gaussian distribution with a mean $L_{m}$ and standard deviation equal to $20 \%$. The mean length was chosen in order to generate a DGD distribution with a mean value around $41 \mathrm{ps}$. The pdfs of first-order PMD, which were obtained for systems with 3,5,10, and 15 PMFs, are shown in Fig. 11(a)-(d), respectively. In Fig. 11(a) and (b), we observe that both three and five PMFs are insufficient to mimic the theoretical distribution of the first-order PMD, i.e., a Maxwellian [22]. Fig. 11(c) and (d) shows that a system with ten and more PMFs can reproduce a large range of first-order PMD values with a statistic close to the Maxwellian distribution, including the tail distribution.

The magnitude distributions of the first- and second-order PMD vectors were compared by a commonly used parameter: the normalized deviation factor (NDF) [15], [16]. Fig. 12 presents the NDF of the first- and second-order PMD, for the cases where the scattering sections comprise one and three PCs,

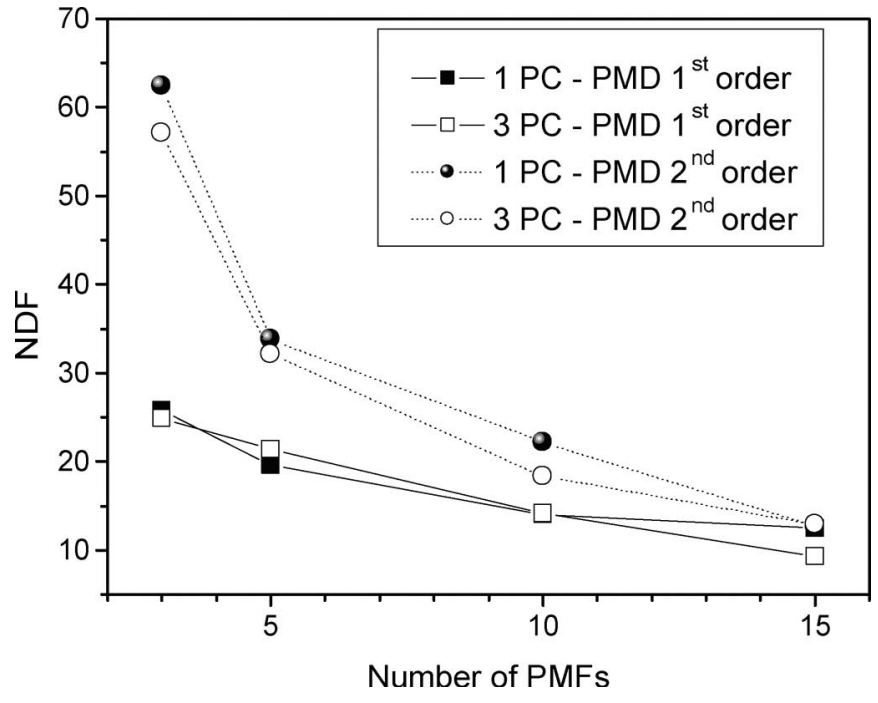

Fig. 12. NDF for first- and second-order PMD as function of the number of PMFs. Scattering sections comprising one and three PCs are represented as solid and open symbols, respectively.

as function of the number of PMFs. In a global analysis, results show that as the number of PMFs increases, the NDF parameter decreases, which is in good agreement with the previous results 

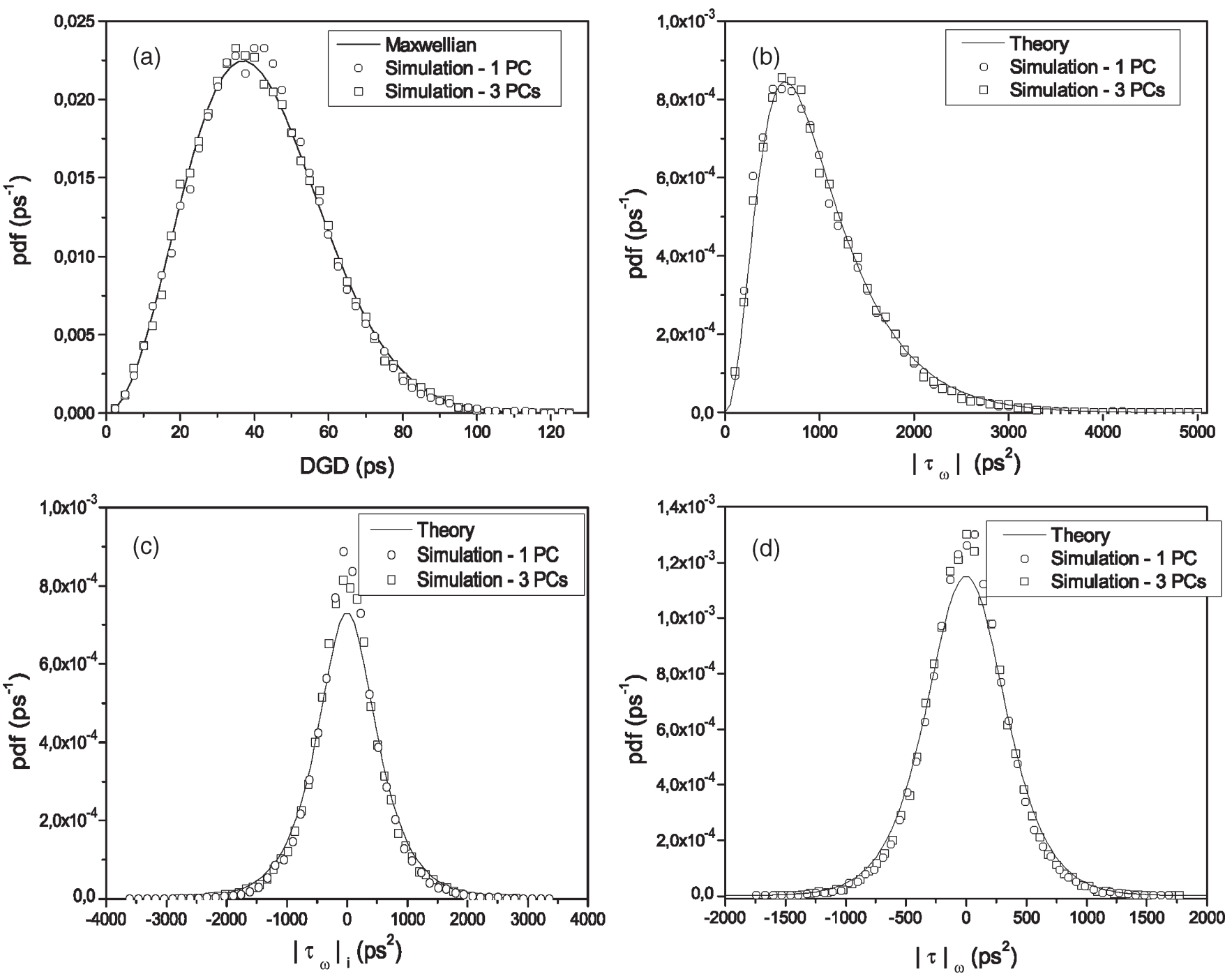

Fig. 13. First- and second-order PMD functions of an emulator with 15 sections. (a) First-order PMD. (b) Magnitude of the second order PMD vector. (c) Component of the second order PMD vector. (d) Second-order component associated with PCD.

presented in [15]. For a fixed number of PMFs, a statistical improvement is, in general, also observed when a more uniform distribution is performed, i.e., when using three PCs instead of just one PC. Nevertheless, the number of PMFs seems to be a more critical parameter when accurate PMD statistics are required.

Fig. 13 represents the pdfs for the emulator with 15 PMFs, with a polarization scattering between PMFs using three and one PCs. The magnitude of the first-order PMD vector is represented in Fig. 13(a). The magnitude and one component of the second-order PMD vector $\vec{\tau}_{\omega}$ are represented in Fig. 13(b) and (c), respectively. The second-order PMD component associated with the polarization-dependent chromatic dispersion (PCD) [23] is represented in Fig. 13(d). The Maxwellian correspondent to the first-order pdf, represented in Fig. 13(a) as a solid line, has a mean value equal to 41 ps. The remaining theoretical pdfs, which are also represented as solid lines, are plotted with the fit parameter equal to the mean value of 41 ps. For both cases (polarization scattering with one and three PCs), the simulation results are in good agreement with the theory. Using these results in conjugation with the ones presented in Fig. 12, we conclude that a large number of PMFs should be considered in an emulator design and that the uniformity of the polarization scattering is a less relevant factor.

\section{CONCLUSION}

In this paper, we have presented a detailed study of fibercoil-based PCs. First, a method to deterministically calculate the PC configuration in order to transform the polarization between any two SOPs was presented. Second, an expression for the mean-square values of the Stokes parameters when several fiber-coil-based PC devices are concatenated was derived. This expression was extensively confirmed with simulation results. We showed that with three PCs, a uniform polarization scattering is obtained. An application of the polarization scattering to PMD emulation was also presented. First- and second-order PMD statistics are well generated when 15 PMFs are interconnected with 14 polarization-scattering sections. We also conclude that with these high numbers of polarization-scattering sections, the number of PCs used 


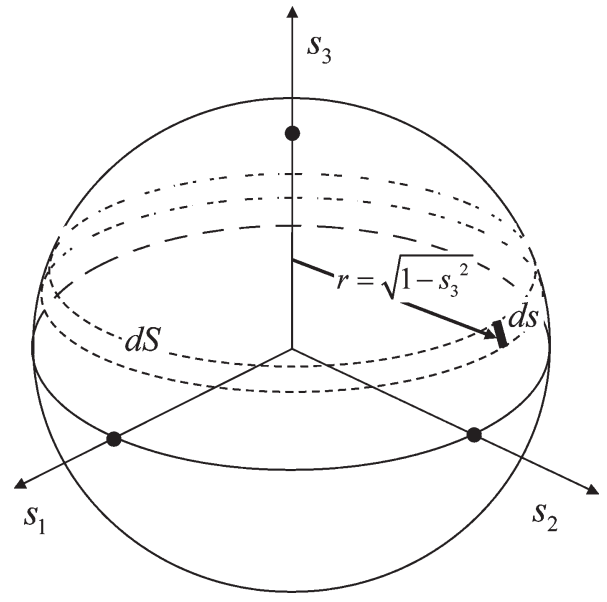

Fig. 14. Poincare sphere representation of the area element of the surface of revolution.

in each section to scatter the light polarization becomes less critical.

\section{APPENDIX \\ UNIFORM DISTRIBUTION OVER THE POINCARÉ SPHERE}

In this Appendix, a uniform distribution of Stokes vectors over the Poincare sphere is considered. The distribution properties of each vector component $s_{i}$ are derived. The area element of a surface revolution obtained around the $s_{3}$ axis is given by

$$
d S=2 \pi r d s
$$

where $d s$ is a curve element, and $r$ is the distance between the $s_{3}$ axis and the sphere surface (see Fig. 14). The $d s$ curve element can be written as

$$
d s^{2}=d r^{2}+d s_{3}^{2}
$$

where $d r$ and $d s_{3}$ are infinitesimal increments on $r$ and $s_{3}$, respectively. Taking the square root, (45) can be written as

$$
d s=\sqrt{1+\left(\frac{d r}{d s_{3}}\right)^{2}} d s_{3} .
$$

The distance $r$ can be written as a function of $s_{3}$

$$
r\left(s_{3}\right)=\sqrt{1-s_{3}^{2}} .
$$

These results can be used to derive $d s$. Taking the derivative of $r$ with respect to $s_{3}$ and using it in (46), we get the following expression for $d s$ :

$$
d s=\frac{d s_{3}}{\sqrt{1-s_{3}^{2}}} .
$$

Using (48) and (47) in (44), we get the following expression for $d S$ :

$$
d S=2 \pi d s_{3} .
$$

Thus, the area $S$ between $s_{3}=a$ and $s_{3}=b$, with $b-a=h$, is just

$$
S=2 \pi \int_{a}^{b} d s_{3}=2 \pi h .
$$

Notice how the integrand reduces to a constant. This proof also works for the $s_{1}$ and $s_{2}$ axes. Using this result, we can conclude that if we have a uniform distribution of points over the sphere surface, the respective projections are also uniformly distributed between -1 and 1 . Therefore, observing the normalization condition, the three coordinate pdfs $g_{s_{i}}$ have the constant value $1 / 2$.

As the mean value of each coordinate is zero, the variances $\sigma_{i}$ are given by the mean squares

$$
\sigma_{i}=\left\langle s_{i}^{2}\right\rangle=\int_{-1}^{1} g_{s_{i}} s_{i}^{2} d s_{i} .
$$

Using $g_{s_{i}}=1 / 2$ in (51), we obtain for the variances the one third value.

\section{REFERENCES}

[1] R. Ulrich, "Polarization stabilization on single-mode fibers," Appl. Phys. Lett., vol. 35, no. 11, pp. 840-842, Dec. 1979.

[2] H. Honmou, S. Yamazaky, K. Emura, R. Ishikawa, I. Mito, M. Shikada, and K. Minemura, "Stabilisation of heterodyne receiver sensitivity with automatic polarisation control system," Electron. Lett., vol. 22, no. 22, pp. 1181-1182, 1986

[3] M. Johnson, "In-line fiber-optical polarization transformer," Appl. Opt., vol. 18 , no. 9 , pp. $1288-1289$, May 1979

[4] P. Granestrand and L. Thylen, "Active stabilization of polarization on a single-mode fiber," Electron. Lett., vol. 20, no. 9, pp. 365-366, Apr. 1984.

[5] H. C. Lefevre, "Single-mode fibre fractional wave devices and polarization controllers," Electron. Lett., vol. 16, no. 20, pp. 778-780, Sep. 1980.

[6] Y. Kidoh, "Polarization control on output of single-mode optical fibers," J. Quantum Electron., vol. QE-, no. 6, pp. 991-994, 1981.

[7] T. Okoshi, Y. H. Cheng, and K. Kikuchi, "New polarization control scheme for optical heterodyne receiver using two faraday rotators," Electron. Lett., vol. 21, no. 18, pp. 787-788, Aug. 1985.

[8] W. L. Barnes, " $2 \times 2$ optical fiber polarization switch and polarization controller," Electron. Lett., vol. 24, no. 23, pp. 1427-1429, 1988.

[9] A. Siddiqui and H. Sherief, "Liquid crystal polarisation controller for use in fiber communication systems," presented at the Optical Fiber Commun. Conf., Houston, TX, Feb. 6-9, 1989.

[10] H. C. LeFevre, presented at the Fiber Optic Polarization Controller Conf., Jun. 21, 1983

[11] B. G. Koehler and J. E. Bowers, "In-line single-mode fiber polarization controllers at 1500, 1300 and $630 \mathrm{~nm}$," Appl. Opt., vol. 24, no. 3, pp. 349-353, Feb. 1985.

[12] N. G. Walker and G. R. Walker, "Polarization control for coherent communications," J. Lightw. Technol., vol. 8, no. 3, pp. 438-458, Mar. 1990.

[13] R. Ulrich, S. C. Rashleigh, and W. Eickhoff, "Bending-induced birefringence in single mode fibers," Opt. Lett., vol. 5, no. 6, pp. 273-275, 1980.

[14] R. Ulrich and A. Simon, "Polarization optics of twisted single-mode fibers," Appl. Opt., vol. 18, no. 13, pp. 2241-2251, Jul. 1979.

[15] I. T. Lima, R. Khosravani, P. Ebrahimi, E. Ibragimov, C. R. Menyuk, and A. E. Willner, "Comparison of polarization mode dispersion emulators," J. Lightw. Technol., vol. 19, no. 12, pp. 1872-1881, Dec. 2001

[16] Y. K. Lizé, L. Palmer, N. Godbout, S. Lacroix, and R. Kashyap, "Scalable polarization-mode dispersion emulator with proper first- and second-order statistics," IEEE Photon. Technol. Lett., vol. 17, no. 11, pp. 2451-2453, Nov. 2005.

[17] D. Marcuse, C. R. Menyuk, and P. K. Wai, "Application of the Manakov-PMD equation to studies of signal propagation in optical fibers with randomly varying birefringence," J. Lightw. Technol., vol. 15, no. 9 , pp. 1735-1746, Sep. 1997. 
[18] N. Gisin and J. P. Pellaux, "Polarization mode dispersion: Time versus frequency domains," Opt. Commun., vol. 89, no. 2/4, pp. 316-323, May 1992.

[19] J. P. Gordon and H. Kogelnik, "PMD fundamentals: Polarization mode dispersion in optical fibers," Proc. Nat. Acad. Sci., vol. 97, no. 9, pp. 4541-4550, 2000.

[20] A. Djupsjöbacka, "Comparison of polarization mode dispersion emulators," J. Lightw. Technol., vol. 19, no. 12, pp. 1872-1881, Dec. 2001.

[21] R. M. Jopson, L. E. Nelson, and H. Kogelnik, "Measurement of secondorder polarization-mode dispersion vectors in optical fibers," IEEE Photon. Technol. Lett., vol. 11, no. 9, pp. 1153-1155, Sep. 1999.

[22] F. Curti, B. Daino, G. D. Marchis, and F. Matera, "Statistical treatment of the evolution of the principal states of polarization in single-mode fibers," J. Lightw. Technol., vol. 8, no. 8, pp. 1162-1166, Aug. 1990.

[23] G. J. Foschini, R. M. J. L. E. Nelson, and H. Kogelnik, "Probability densities of second-order polarization mode dispersion including polarization dependent chromatic fiber dispersion," IEEE Photon. Technol. Lett., vol. 12, no. 3, pp. 293-295, Mar. 2000.

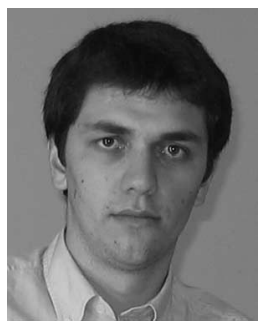

Nelson J. Muga was born in Mogadouro, Portugal, in 1980. He received the physics degree from University of Porto, Porto, Portugal, in 2002 and the M.S. degree in applied physics from University of Aveiro, Aveiro, Portugal, in 2006 where he is currently working toward the Ph.D. degree.

His current main interest of research is polarization effects in optical fibers.

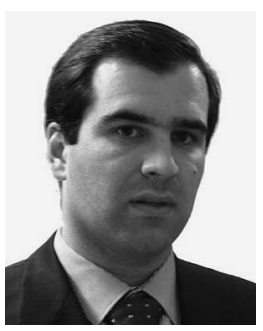

Armando Nolasco Pinto (M'00) was born in Oliveira do Bairro, Portugal, on 1971. He received the degree in electronic and telecommunications engineering and the Ph.D. degree in electrical engineering, both from the University of Aveiro, Aveiro, Portugal, in 1994 and 1999, respectively.

$\mathrm{He}$ is currently an Assistant Professor with the Electrical and Telecommunications Department, University of Aveiro, and a Researcher with the Institute of Telecommunications, Aveiro. He is the author and/or coauthor of more than 60 research papers. His main research interests focus on optical communication systems, mainly in nonlinear, polarization, and temperature-dependent effects in fiber optics.

Dr. Pinto is a member of the Optical Society of America (OSA) and the IEEE Lasers and Electro-Optics Society (LEOS).

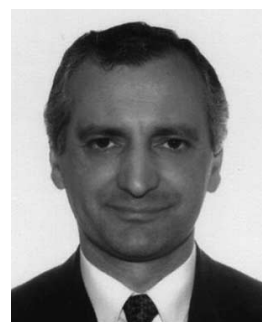

Mário F. S. Ferreira was born in Ovar, Portugal. He received the degree in physics from the University of Porto, Porto, Portugal, in 1984, and the Ph.D. degree in physics from the University of Aveiro, Aveiro, Portugal, in 1992

Currently, he is an Associate Professor with the Physics Department, University of Aveiro, where he leads a research group dedicated to the modeling and characterization of multisection semiconductor lasers for coherent systems, quantum well lasers, optical fiber amplifiers and lasers, nonlinear effects, and soliton propagation in optical fibers. He has written more than 150 papers for scientific journals and conference publications, as well as the book Optics and Photonics (in Portuguese).

Dr. Ferreira is a member of the Optical Society of America (OSA), SPIE-The International Society for Optical Engineering, The New York Academy of Sciences (NYAS), the American Association for the Advancement of Science (AAAS), the European Optical Society (EOS), the European Physical Society (EPS), and the Portuguese Physical Society. He was a member of the Editorial Advisory Committee of Optics and Photonics News (OSA) during 1999, he is presently an Associate Editor of Optical Fiber Technology-Materials, Devices, and Systems (Elsevier), and a member of the Advisory Board of Fiber and Integrated Optics (Taylor \& Francis) and Nonlinear Optics (Old City Publishing).

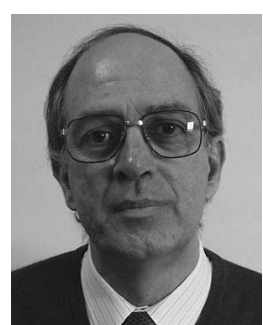

José R. Ferreira da Rocha (M'84) was born in Mozambique. He received the M.Sc. degree in telecommunication systems and the Ph.D. degree in electrical engineering both from University of Essex, Essex, U.K., in 1980 and 1983, respectively.

He participates actively with the Telecommunications Institute, which is a national R\&D nonprofit organization, where he is a member of the Management Committee (Aveiro, Portugal branch) and the National Coordinator for the optical communications area. He has coordinated the University of Aveiro and Telecommunications Institute participation in various projects included in the following European Union (EU) R\&D Programs in the area of telecommunications: RACE, RACE II, ACTS, and IST. Over the past few years, he has acted as a Technical Auditor, Evaluator, and Independent Observer for the evaluation of projects submitted to various EU R\&D Programs. He has also participated in various project evaluation boards set up by the Engineering and Physical Sciences Research Council (EPSRC), United Kingdom. By invitation of the ACTS Management Committee, he participated in the "Expert Groups on Visionary Research in Communications," which aimed to create a bridge between the activities carried out in the Fourth and the Fifth Framework Programs on EU activities in the field of research, technological development, and demonstration. He is currently a Full Professor with the University of Aveiro. He has published about 170 papers, mainly in international journals and conferences. His present research interests include modulation formats and receiver design for very high-capacity (above $40 \mathrm{~Gb} / \mathrm{s}$ ) optical communication systems based on linear and nonlinear transmission and wavelength division multiplexing optical networks. 